\title{
Study on Indefinite Stochastic Linear Quadratic Optimal Control with Inequality Constraint
}

\author{
Guiling $\mathrm{Li}^{1}$ and Weihai Zhang ${ }^{2}$ \\ ${ }^{1}$ College of Information Science and Engineering, Shandong University of Science and Technology, Qingdao 266591, China \\ ${ }^{2}$ College of Information and Electrical Engineering, Shandong University of Science and Technology, Qingdao 266591, China \\ Correspondence should be addressed to Weihai Zhang;w_hzhang@163.com
}

Received 1 April 2013; Revised 12 September 2013; Accepted 2 October 2013

Academic Editor: Lotfollah Najjar

Copyright (c) 2013 G. Li and W. Zhang. This is an open access article distributed under the Creative Commons Attribution License, which permits unrestricted use, distribution, and reproduction in any medium, provided the original work is properly cited.

\begin{abstract}
This paper studies the indefinite stochastic linear quadratic (LQ) optimal control problem with an inequality constraint for the terminal state. Firstly, we prove a generalized Karush-Kuhn-Tucker (KKT) theorem under hybrid constraints. Secondly, a new type of generalized Riccati equations is obtained, based on which a necessary condition (it is also a sufficient condition under stronger assumptions) for the existence of an optimal linear state feedback control is given by means of KKT theorem. Finally, we design a dynamic programming algorithm to solve the constrained indefinite stochastic LQ issue.
\end{abstract}

\section{Introduction}

The study on LQ control problems can be traced back to the pioneering work of Kalman [1] and Wonham [2] several decades ago. The LQ control theory is elegantly established and developed, and the main work can be seen in [3-11]. In particular, it is found [6] that a stochastic LQ problem with indefinite control weighting matrices may still be well-posed. This discovery evokes a series of subsequent researches, and many important achievements are obtained [5, 12-16]. Up to now, most work deals with the indefinite stochastic LQ problems without constraints. However, as a practical optimization problem, the indefinite stochastic LQ problem unavoidably has various constraints on the state or control; in particulary the inequality constraints often appear.

For the constrained indefinite stochastic LQ problems, [17] studied the equally constrained stochastic LQ optimization for Itô systems. In this paper, we will study the stochastic LQ problem with inequality constraint.

Firstly, we present and prove the generalized KKT theorem under hybrid constraints. Secondly, a necessary condition for the existence of an optimal linear state feedback control is given by means of the generalized KKT theorem. Thirdly, if we strengthen the condition, we can obtain a necessary and sufficient condition for the existence of the optimal linear feedback control to indefinite stochastic LQ optimal control problem with inequality constraint. Finally, we give a dynamic programming algorithm to solve the stochastic LQ problem with the inequality constraint. We provide an example to demonstrate the effectiveness of our main theoretical results.

The outline of this paper is organized as follows. In Section 2, we present a generalized KKT theorem under hybrid constraints. Section 3 proposes a KKT condition for the existence of an optimal linear state feedback control. In Section 4, we provide a necessary and sufficient condition and a dynamic programming algorithm for the stochastic LQ problem with inequality constraint. Section 5 concludes the paper.

For convenience, throughout the paper, we adopt the following notations: $A^{T}$ denotes the transpose of a matrix $A$. $A>0(A \geq 0): A$ is a positive definite (positive semidefinite) symmetric matrix. $\operatorname{tr}(A)$ : trace of a square matrix. $R^{m \times n}$ : the space of all $m \times n$ real matrices. $S^{n}$ : a $n \times n$ symmetric matrix space.

\section{Preliminaries}

Consider the following indefinite stochastic LQ control. 
Problem 1. Consider

$$
\begin{gathered}
\min \quad J\left(x_{0}, u\right)=E \int_{0}^{T}\left[x^{T}(t) Q(t) x(t)\right. \\
\\
\left.+u^{T}(t) R(t) u(t)\right] d t, \\
\text { s.t. } \quad d x(t)=[A(t) x(t)+B(t) u(t)] d t \\
+[C(t) x(t)+D(t) u(t)] d w(t), \\
x(0)=x_{0}, \\
E\left\{\|x(T)\|^{2}\right\}=E\left[x^{T}(T) x(T)\right] \leq c,
\end{gathered}
$$

where $x(t)=\left[x_{1}(t), x_{2}(t), \ldots, x_{n}(t)\right]^{T}$ is an $n$-dimensional state variable, $u(t) \in R^{m}$ is a control input, $w(\cdot)$ is a onedimensional standard Brownian motion defined on a filtered probability space $\left(\Omega, F, F_{t}, P\right)$. We denote the information flow $\mathscr{F}_{t}=\sigma[w(s): 0 \leq s \leq t \leq T] . u(\cdot)$ belongs to $\mathscr{L}_{\mathscr{F}}^{2}\left(R^{m}\right)$, where $\mathscr{L}_{\mathscr{F}}^{2}\left(R^{m}\right)$ is a space of all $R^{m}$-valued, $\mathscr{F}_{t}$-adapted measurable processes satisfying $E \int_{0}^{T}\|u(t)\|^{2} d t<+\infty$. For each admissible control, the corresponding trajectory satisfies the constraint (1c). $c$ in constraint (1c) is a given nonnegative constant. $A(t), B(t), C(t), D(t), Q(t)$, and $R(t)$ are timevarying matrices of suitable dimensions. $Q(t)$ and $R(t)$ in objective functional are symmetric matrices. To study the issue, we first put forward the following Assumption $\mathrm{H}_{1}$.

Assumption $H_{1} . A(t), C(t) \in \mathscr{L}^{\infty}\left(0, T, R^{n \times n}\right), Q(t) \in \mathscr{L}^{\infty}(0$, $\left.T, S^{n}\right), B(t), D(t) \in \mathscr{L}^{\infty}\left(0, T, R^{n \times m}\right)$, and $R(t) \in \mathscr{L}^{\infty}(0, T$, $\left.S^{m}\right)$, where $L^{\infty}(0, T, X):=\{f(t): X$-valued essential bounded measurable function and ess $\left.\sup _{t \in[0, T]}\|f(t)\|<+\infty\right\}$.

In this paper, the weighting matrices in the objective functional are not required to be definite. Therefore Problem 1 is an indefinite stochastic LQ optimal control problem. For later use, we recall KKT theorem for this type of mathematical programming (MP) problems:

$$
\begin{array}{ll}
\min & f(x) \\
\text { s.t. } & \mathbf{g}(x) \leq \mathbf{0}, \\
& \mathbf{h}(x)=\mathbf{0},
\end{array}
$$

where $\mathbf{g}(x)=\left(g_{1}(x), \ldots, g_{p}(x)\right)$, and $\mathbf{h}(x)=\left(h_{1}(x), \ldots, h_{q}(x)\right)$.

The KKT conditions [18-20], which are also known as the Kuhn-Tucker (KT) conditions, are the first-order necessary conditions for a solution in nonlinear programming to be optimal, provided that some regularity conditions are satisfied. The Lagrange multiplier method, which allows only equality constraints, can be viewed as a special case of KKT conditions.

Regularity Condition (or Constraint Qualification). In MP above, let $I^{*}=\left\{i \mid g_{i}\left(x^{*}\right)=0\right\}\left(g_{i}\left(x^{*}\right) i \in I^{*}\right.$ be active constraints at $\left.x^{*}\right)$. The gradient vectors $\nabla g_{i}\left(x^{*}\right), i \in I^{*}$, and $\nabla h_{j}\left(x^{*}\right), j=1, \ldots, q$, are linearly independent, is known as a linear independent constraint qualification (LICQ).

Regular Point. In MP above, $x^{*}$ is said to be a regular point of the constraints if the gradient vectors $\nabla g_{i}\left(x^{*}\right), \nabla h_{j}\left(x^{*}\right), i \in$ $I^{*}, j=1, \ldots, q$, are linearly independent.

KKT Theorem. In MP above, we assume that the functions $f, \mathbf{g}=\left(g_{1}, \ldots, g_{p}\right)^{T}, \mathbf{h}=\left(h_{1}, \ldots, h_{q}\right)^{T}$ are twice continuously differentiable and we assume that all the constraints satisfy the regularity condition LICQ. Let $x^{*}$ be a point satisfying all the constraints and let $x^{*}$ be a regular point of the above constraints. Now suppose that this regular point $x^{*}$ is also a relative minimum point for the original MP. Then it is shown that there exist a vector $\lambda \geq 0 \in R$ and a vector $\mu \in R$, such that

$$
\begin{gathered}
\nabla_{x} L\left(x^{*}, \lambda^{*}, \mu^{*}\right)=\mathbf{0}, \\
\lambda_{i}^{*} g_{i}\left(x^{*}\right)=0, \quad i=1, \ldots, p,
\end{gathered}
$$

where $L(x, \lambda, \mu)=f(x)+\lambda^{T} \mathbf{g}(x)+\mu^{T} \mathbf{h}(x)$ is the Lagrangian function and $\lambda_{i}^{*} g_{i}\left(x^{*}\right)=0, i=1, \ldots, p$, are complementary clackness condition.

It is particularly important to check the regularity condition before we apply the conclusion of KKT theorem. If it is not so, the conclusion of KKT theorem would not be valid, just as the following example shows.

Example 2. Consider

$$
\begin{array}{ll}
\min & f\left(x_{1}, x_{2}\right)=-2 x_{1}-3 x_{2}, \\
\text { s.t. } & \mathbf{g}\left(x_{1}, x_{2}\right)=x_{1}^{2}+x_{2}^{2}=0 .
\end{array}
$$

Obviously, the minimum point is $x^{*}(0,0)^{T}$. According to KKT theorem, we obtain

$$
\begin{gathered}
2-\lambda x_{1}^{*}=0, \\
3-\lambda x_{2}^{*}=0, \\
x_{1}^{* 2}+x_{2}^{* 2}=0 .
\end{gathered}
$$

The conclusion of KKT theorem does not hold at point $x^{*}(0,0)^{T}$, because $\nabla \mathbf{g}(0,0)=(0,0)^{T}$ is not linearly independent. It does not satisfy the LICQ regularity condition.

In order for a minimum point $x^{*}$ to satisfy the above KKT conditions, it should satisfy some regularity conditions. Except for LICQ regularity condition, the most used ones are listed below.

Constant Rank Constraint Qualification. For each subset of the gradients of the active inequality constraints and the gradients of the equality constraints the rank at a vicinity of $x^{*}$ is constant.

Mangasarian-Fromovitz Constraint Qualification. The gradients of the active inequality constraints and the gradients of the equality constraints are linear independent at $x^{*}$.

Constant Positive Linear Dependence Constraint Qualification. For each subset of the gradients of the active inequality 
constraints and the gradients of the equality constraints, if it is positive-linear dependent at $x^{*}$, then it is positive-linear dependent at a vicinity of $x^{*}$.

The Slater condition for a convex MP is also a common regularity condition.

Remark 3. In this paper, for convenience, when we use the KKT theorem, we always assume that the local optimal $x^{*}$ meets the LICQ regularity condition. The same goes for other regularity conditions.

Definition 4 (see [21]). Let $X$ be a vector space, $Y$ a normed space, and $T$ a transformation from $X$ to $Y$. If the limit

$$
\delta T(x ; h)=\lim _{\alpha \rightarrow 0} \frac{[T(x+\alpha h)-T(x)]}{\alpha}
$$

exists, it is called the Gateaux differential of $T$ at $x$ with increment $h$. If the limit exists for each $h \in X$, the transformation $T$ is said to be Gateaux differentiable at $x$.

Definition 5 (see [21]). Let $X$ be a vector space and $Z$ a Banach space with a positive cone $P$ having nonempty interior. Let $G$ be a mapping from $X$ to $Z$ which has a Gateaux differential that is linear in its increment. A point $x_{0} \in X$ is said to be a regular point of the inequality $G(x) \leq 0$, if $G\left(x_{0}\right) \leq 0$ and there is an $h \in X$ such that $G\left(x_{0}\right)+\delta G\left(x_{0} ; h\right)<0$.

Definition 6 (see [21]). Let $X$ be a vector space and $Z$ a Banach space. Let $H(x)=\left[h_{1}(x), \ldots, h_{1}(x)\right]^{T}$ be a mapping from $X$ to $Z$ which has a Gateaux differential that is linear in its increment. A point $x_{0} \in X$ is said to be a regular point of the equality $H(x)=0$, if $\delta h_{1}\left(x_{0} ; h\right), \ldots, \delta h_{n}\left(x_{0} ; h\right)$ are linearly independent.

On the basis of the definitions above, let us discuss the KKT theorem in Banach space, where the objective function and the constraint functions in MP are functionals.

Let us consider

$$
\begin{array}{ll}
\min & f(x) \\
\text { s.t. } & G(x) \leq 0 \\
& H(x)=0 .
\end{array}
$$

As a special case,

$$
\begin{array}{ll}
\min & f(x) \\
\text { s.t. } & G(x) \leq 0
\end{array}
$$

has the local necessary condition as follows.

Lemma 7 (see [21] (generalized KKT theorem)). Let $X$ be a vector space and $Z$ a Banach space having positive cone $P$. Assume that $P$ contains an interior point. Let $f$ be a Gateaux differentiable functional on $X$ and $G$ a Gateaux differentiable mapping from $X$ to $Z$. Assume that the Gateaux differentials are linear in their increments. Suppose that $x_{0}$ minimizes $f$ subject to $G(x) \leq 0$ and that $x_{0}$ is a regular point of the inequality $G(x) \leq 0$. Then there is a $z_{0}^{*} \geq 0$ in $Z$, such that the Lagrangian function $f(x)+z_{0}^{*} G(x)$ is stationary at $x_{0}$. Furthermore $z_{0}^{*} G\left(x_{0}\right)=0$. $(M P I)$

The following theorem is the local necessary condition of

Theorem 8. Let $X$ be a vector space and $Z$ a Banach space having positive cone $P$. Assume that $P$ contains an interior point. Let $f$ be a Gateaux differentiable functional on $X$. Let $G$ and $H$ be Gateaux differentiable mappings from $X$ to $Z$. Assume that the Gateaux differentials are linear in their increments. Suppose that $x_{0}$ minimizes $f$ subject to $G(x) \leq 0$, $H(x)=0$ and that $x_{0}$ is a regular point of $G(x) \leq 0, H(x)=0$. Then there is a $\lambda^{*} \geq 0$ in $Z, \mu^{*} \in Z$, such that the Lagrangian function $f(x)+\lambda^{*} G(x)+\mu^{*} H(x)$ is stationary at $x_{0}$. Namely, $\delta f\left(x_{0} ; h\right)+\lambda^{*} \delta G\left(x_{0} ; h\right)+\mu^{*} \delta H\left(x_{0} ; h\right)=0$. Furthermore, $z_{0}^{*} G\left(x_{0}\right)=0$.

Proof. $H(x)=0$ is equivalent to $H(x) \leq 0$ and $-H(x) \leq$ 0 . If $x_{0}$ is a regular point of $H(x)=0$, then $\delta h_{1}\left(x_{0} ; h\right), \ldots$, $\delta h_{n}\left(x_{0} ; h\right)$ are linearly independent. So $\delta h_{1}\left(x_{0} ; h\right), \ldots$, $\delta h_{n}\left(x_{0} ; h\right)$ are all nonzero, because the Gateaux differentials are linear in their increments. Using Definition 5, it is easy to verify that $x_{0}$ is a regular point of both $-H(x) \leq 0$ and $H(x) \leq 0$. According to Lemma 7, we know that the multiplier $\mu^{*}$ of equality $H(x)=0$ has no nonnegative requirement.

Definition 9 (see [22]). Suppose that $f(X)$ is a scalar-valued function of the elements $x_{i j}$ of $X$. Then the gradient matrix of $f(X)$ is defined as

$$
\nabla[f(X)]=\frac{\partial f(X)}{\partial(X)}
$$

with

$$
\left[\frac{\partial f(X)}{\partial(X)}\right]_{i j}=\frac{\partial f(X)}{\partial x_{i j}} .
$$

Based on Definition 9, we can easily extend KKT theorem from Banach space to matrix space. Because $X(t)$ can be treated as a vector $\left[X_{1}(t), \ldots, X_{n}(t)\right]^{T}$, one can work out $\nabla\left[X_{i}(t)\right]$ and the KKT theorem holds.

When we apply the matrix KKT theorem, we need to give the partial list of gradient matrices [22] that we will use in this paper. In the following formulas, $X$ is an $n \times m$ matrix. The formulas are not valid if the elements $x_{i j}$ of $X$ are not independent. $A, B$ are assumed to have appropriate dimensions determined from context.

Consider the following:

$$
\begin{gathered}
\frac{\partial}{\partial X} \operatorname{tr}(X)=I, \\
\frac{\partial}{\partial X} \operatorname{tr}\left(X X^{T}\right)=2 X, \\
\frac{\partial}{\partial X} \operatorname{tr}\left(A X^{T}\right)=A, \\
\frac{\partial}{\partial X} \operatorname{tr}(A X)=A^{T},
\end{gathered}
$$




$$
\begin{aligned}
& \frac{\partial}{\partial X} \operatorname{tr}\left(A X^{T} B\right)=B A \\
& \frac{\partial}{\partial X} \operatorname{tr}(A X B)=A^{T} B^{T} \\
& \frac{\partial}{\partial X} \operatorname{tr}\left(A X B X^{T}\right)=A^{T} X B^{T}+A X B \\
& \frac{\partial}{\partial X} \operatorname{tr}(A X B X)=A^{T} X^{T} B^{T}+B^{T} X^{T} A^{T}
\end{aligned}
$$

\section{KKT Conditions and a New Type of GDREs}

Definition 10. Problem 1 is well-posed, if for any $x_{0} \in R^{n}$, $-\infty<V\left(x_{0}\right)=\inf _{u(\cdot) \in U_{a d}}\left\{J\left(x_{0}, u(\cdot)\right)\right\} . u_{*}$ is called an optimal control, if $V\left(x_{0}\right)=J\left(x_{0}, u_{*}\right)$, and $x_{*}$ denotes the corresponding optimal trajectory.

Let the control law be $u=K x\left(K \in C^{m \times n}[0, T]\right)$, and $X(t)=E\left[x(t) x^{T}(t)\right]$. By substituting $u=K x$ into (1a) of Problem 1, we obtain the new objective functional:

$$
J(X, K)=\operatorname{tr} \int_{0}^{T}\left[Q X+K^{T} R K X\right] d t,
$$

where $C^{n \times n}[0, T]$ is the space of $n$-order square matrix whose elements are continuous functions. By substituting $u=K x$ into (1b) of Problem 1, we obtain a closed-loop system:

$$
\begin{aligned}
d x= & (A+B K) x d t \\
& +(C+D K) x d w, \quad x(0)=x_{0} .
\end{aligned}
$$

By applying Itô's formula to $X(t)$, we obtain

$$
\begin{gathered}
\dot{X}=(A+B K) X+X(A+B K)^{T} \\
+(C+D K) X(C+D K)^{T} \\
X(0)=X_{0}=x_{0} x_{0}^{T} .
\end{gathered}
$$

Define the transformation $H(X, K)$ from $C^{n \times n}[0, T] \times C^{m \times n}$ $[0, T]$ to $C^{n \times n}[0, T]$ :

$$
\begin{aligned}
H(X, K):= & X(t)-X(0) \\
& -\int_{0}^{t}\left[(A+B K) X+X(A+B K)^{T}\right. \\
& \left.+(C+D K) X(C+D K)^{T}\right] d t .
\end{aligned}
$$

By substituting $u=K x$ into (1c) of Problem 1, we obtain

$$
\operatorname{tr} X(T)-c \leq 0
$$

Define the transformation $G(X, K)$ from $C^{n \times n}[0, T]$ to $R^{1}$ :

$$
G(X(T)):=\operatorname{tr} X(T)-c .
$$

So the original stochastic Problem 1 can be transformed into the deterministic Problem 11 as follows.
Problem 11. Consider the following:

$$
\begin{array}{ll}
\min & J(X, K)=\operatorname{tr} \int_{0}^{T}\left[Q X+K^{T} R K X\right] d t \\
\text { s.t. } & H(X, K)=0, \quad \forall t \in[0, T] \\
& G(X(T)) \leq 0 .
\end{array}
$$

Lemma 12. $J(X, K), H(X, K)$, and $G(X(T))$ have continuous Gateaux derivative as follows:

$$
\begin{gathered}
\delta J_{X}(X, K ; \Delta X)=\operatorname{tr} \int_{0}^{T}\left(Q+K^{T} R K\right) \Delta X d t, \\
\delta J_{K}(X, K ; \Delta X)=\operatorname{tr} \int_{0}^{T}\left(\Delta K^{T} R K X+K^{T} R \Delta K X\right) d t, \\
\delta H_{X}(X, K ; \Delta X)(t) \\
=\Delta X(t)-\operatorname{tr} \int_{0}^{t}\left[(A+B K) \Delta X+\Delta X(A+B K)^{T}\right. \\
\left.+(C+D K) \Delta X(C+D K)^{T}\right] d t,
\end{gathered}
$$

$$
\begin{gathered}
\delta H_{K}(X, K ; \Delta K)(t) \\
=-\operatorname{tr} \int_{0}^{t}\left[B \Delta K X+X \Delta K^{T} B^{T}+(C+D K) X(D \Delta K)^{T}\right. \\
\left.+(D \Delta K) X(C+D K)^{T}\right] d t \\
\delta G_{X}(X ; \Delta X(T))=\operatorname{tr} \Delta X(T) .
\end{gathered}
$$

Proof. We prove only the most complicated one. The rest can be verified in the same way. From Definition 4,

$$
\begin{aligned}
& \delta H_{X}(X, K ; \Delta X)=\lim _{\alpha \rightarrow 0} \frac{[H(X+\alpha \Delta X, K)-H(X, K)]}{\alpha}, \\
& \begin{array}{r}
H(X, K) \\
:=X(T)-X(0)-\int_{0}^{t}\left[(A+B K) X+X(A+B K)^{T}\right. \\
\left.+(C+D K) X(C+D K)^{T}\right] d t .
\end{array}
\end{aligned}
$$

Replace the $X$ in $H(X, K)$ with $(X+\alpha \Delta X)$ and then let $\alpha \rightarrow 0$ showing the conclusion.

Lemma 13 (see [21]). If $\alpha(t)$ and $\beta(t)$ are continuous in $\left[t_{1}, t_{2}\right]$ and $\int_{t_{1}}^{t_{2}}[\alpha(t) h(t)+\beta(t) h(t)] d t=0$ for every continuously differentiable $h(t)$ with $h\left(t_{1}\right)=h\left(t_{1}\right)=0$, then $\beta$ is differentiable and $\alpha(t) \equiv \beta(t)$ in $\left[t_{1}, t_{2}\right]$. 
Lemma 14 (see [21]). If $\alpha(t)$ is continuous in $\left[t_{1}, t_{2}\right]$ and $\int_{t_{1}}^{t_{2}}[\alpha(t) h(t)] d t=0$ for every continuously differentiable $h(t)$ with $h\left(t_{1}\right)=h\left(t_{1}\right)=0$, then $\alpha(t) \equiv 0$ on $\left[t_{1}, t_{2}\right]$.

Theorem 15. Assume that $K_{*}$ is the optimal solution of Problem 1 , and then there exist a symmetric matrix $P \in N B V^{n \times n}$ $[0, T]$ and a nonnegative $\lambda \in R^{1}$, such that

$$
\begin{gathered}
-\dot{P}=\left(Q+K_{*}^{T} R K_{*}\right)+P\left(A+B K_{*}\right)+\left(A+B K_{*}\right)^{T} P \\
+\left(C+D K_{*}\right) P\left(C+D K_{*}\right)^{T}, \\
P(T)=\lambda I_{n}, \\
K_{*}^{T} R+P B+\left(C+D K_{*}\right)^{T} P D=0, \\
\lambda[\operatorname{tr} X(T)-c]=0,
\end{gathered}
$$

where $N B V^{n \times n}[0, T]$ is a matrix space whose elements are bounded functions in $[0, T]$ with 0 value at point 0 and right continuous at $(0, T)$.

Proof. $K_{*}$ is also the optimal solution of Problem 11. Problem 11 is the type of (MP I). Assume that the optimal solution to Problem 11 is $\left(X_{*}, K_{*}\right)$. Using Theorem 8 and Lemma 12, there exist a symmetric matrix $P \in N B V^{n \times n}[0, T]$ and a nonnegative $\lambda \in R^{1}$, such that

$$
\begin{gathered}
\operatorname{tr} \int_{0}^{T}\left(Q+K_{*}^{T} R K_{*}\right) \Delta X d t \\
+\operatorname{tr} \int_{0}^{T}\{\Delta X(t) \\
-\int_{0}^{t}\left[\left(A+B K_{*}\right) \Delta X+\Delta X\left(A+B K_{*}\right)^{T}\right. \\
\left.\left.+\left(C+D K_{*}\right) \Delta X\left(C+D K_{*}\right)^{T}\right] d t\right\} d P \\
+\lambda \operatorname{tr}[\Delta X(T)]=0
\end{gathered}
$$$$
\operatorname{tr} \int_{0}^{T}\left(\Delta K^{T} R K_{*} X_{*}+K_{*}^{T} R \Delta K K_{*}\right) d t
$$$$
-\operatorname{tr} \int_{0}^{T}\left\{\int _ { 0 } ^ { t } \left[B \Delta K X_{*}+X_{*} \Delta K^{T} B^{T}\right.\right.
$$$$
+\left(C+D K_{*}\right) X_{*}(D \Delta K)^{T}+(D \Delta K) X_{*}
$$$$
\left.\left.\times\left(C+D K_{*}\right)^{T}\right] d t\right\} d P=0,
$$

$$
\lambda[\operatorname{tr} X(T)-c]=0 .
$$

For all $(\Delta X, \Delta K) \in C^{n \times n}[0, T] \times C^{m \times n}[0, T],(20)$-(21) are established. According to Riesz representation theorem, we obtain the second item of (20) and the same of (21).

Without loss of generality, let $p(T)=0$. Integrate (20) by parts yielding

$$
\begin{gathered}
\operatorname{tr} \int_{0}^{T}\left(Q+K_{*}^{T} R K_{*}\right) \Delta X d t+\operatorname{tr} \int_{0}^{T} \Delta X d P \\
+\operatorname{tr} \int_{0}^{T}\left\{P \left[\left(A+B K_{*}\right) \Delta X+\Delta X\left(A+B K_{*}\right)^{T}\right.\right. \\
\left.\left.+\left(C+D K_{*}\right)^{T} \Delta X\left(C+D K_{*}\right)^{T}\right]\right\} d t \\
+\lambda \operatorname{tr}[\Delta X(T)]=0 .
\end{gathered}
$$

Clearly, $P$ has no jump on $[0, T)$. But $P$ has a jump at $T$, and the value is $-\lambda I_{n}$. Because the above results are established for all continuous $\Delta X$, then

$$
\int_{0}^{T} \Delta X d P=\left.P \Delta X\right|_{0} ^{T}-\int_{0}^{T} P \Delta \dot{X} d t=-\int_{0}^{T} P \Delta \dot{X} d t .
$$

Thus, (20) becomes

$$
\begin{aligned}
\operatorname{tr} \int_{0}^{T}\left[\left(Q+K_{*}^{T} R K_{*}\right) \Delta X+P\left(A+B K_{*}\right) \Delta X\right. \\
\quad+\left(A+B K_{*}\right)^{T} P \Delta X+\left(C+D K_{*}\right)^{T} P\left(C+D K_{*}\right)^{T} \Delta X \\
\quad-P \Delta \dot{X}] d t=0 .
\end{aligned}
$$

From Lemma $13, P$ is differential in $[0, T)$ and $(19 a)$ is obtained.

In the same way, integrating (21) by parts, we obtain

$$
\begin{aligned}
\operatorname{tr} \int_{0}^{T}[ & K_{*}^{T} R\left(\Delta K X_{*}\right)+P B\left(\Delta K X_{*}\right) \\
& \left.+\left(C+D K_{*}\right)^{T} P D\left(\Delta K X_{*}\right)\right] d t=0 .
\end{aligned}
$$

From Lemma 14, $(19 \mathrm{c})$ is obtained.

To ensure the continuity of $P$, replace $P(T)=0$ with $P(T)$ $=\lambda I_{n}$ (i.e., (19b)). tions.

Equation (19d) is called complementary slackness condi-

Remark 16. Equations (16b)-(16c) of Problem 11 and (19a)(19c) of Theorem 15 are $2 n$-dimensional, first-order differential equations including $2 n$ terminal conditions and $m$ algebraic equations. Equation (19d) is called a complementary slackness condition. By using these conditions, $X_{*}, K_{*}, P$, and $\lambda$ are obtained.

Remark 17. As for the complementary slackness condition, if the inequality constraint of Problem 11 is strict, then $\lambda=0$, and the problem becomes easier. If the inequality constraint of Problem 11 is an equality constraint, it simplifies Theorem 15 as Lagrange multiplier method. 
Definition 18. $M \in R^{n \times n}$ is a given matrix. One calls $M^{+}$the Moore-Penrose generalized inverse of $M$, if

$$
\begin{array}{cc}
M M^{\dagger} M=M, & M^{\dagger} M M^{\dagger}=M^{\dagger}, \\
\left(M M^{\dagger}\right)^{T}=M M^{\dagger}, & \left(M^{\dagger} M\right)^{T}=M^{\dagger} M .
\end{array}
$$

Based on Definition 18, we can rewrite Theorem 15 by expressing $K_{*}$ in terms of $P$.

Lemma 19 (see [13]). Let matrices $L, M$, and $N$ be given with appropriate sizes. Then the matrix equation

$$
L X M=N
$$

has a solution $X$ if and only if

$$
L L^{\dagger} N M^{\dagger} M=N \text {. }
$$

Moreover, any solution to $L X M=N$ is represented by

$$
X=L^{\dagger} N M^{\dagger}+S-L^{\dagger} L S M M^{\dagger}
$$

where $S$ is a matrix with an appropriate size.

Theorem 20. If $K_{*}$ is optimal solution of Problem 1 , then there exist a unique $P \in N B V^{n \times n}[0, T]$ and a nonnegative $\lambda \in R^{1}$, such that

$$
\begin{gathered}
-\dot{P}=\left(Q+K_{*}^{T} R K_{*}\right)+P\left(A+B K_{*}\right) \\
+\left(A+B K_{*}\right)^{T} P+\left(C+D K_{*}\right) P\left(C+D K_{*}\right)^{T} \\
P(T)=\lambda I_{n} \\
K_{*}^{T} R+P B+\left(C+D K_{*}\right)^{T} P D=0, \\
\lambda[\operatorname{tr} X(T)-c]=0 \\
K_{*}=-\left(R+D^{T} P D\right)^{\dagger}\left(B^{T} P+D^{T} P C\right) \\
+Y-\left(R+D^{T} P D\right)^{\dagger}\left(R+D^{T} P D\right) Y
\end{gathered}
$$

where $Y \in L^{2}\left(0, T ; R^{m \times n}\right)$.

Proof. Form (19c) in Theorem 15, we obtain

$$
\left(R+D^{T} P D\right) K_{*}=-\left(B^{T} P+D^{T} P C\right) .
$$

According to Lemma 19,

$$
\begin{aligned}
K_{*}(R & \left.+D^{T} P D\right)^{+}\left(B^{T} P+D T P C\right)+Y \\
& -\left(R+D^{T} P D\right)^{+}\left(B^{T} P+D^{T} P C\right) Y
\end{aligned}
$$

where $Y \in L^{2}\left(0, T ; R^{m \times n}\right)$.

As a special case, let us consider the following discrete stochastic LQ control problem without inequality constraint.
Problem 21. Consider the following.

$$
\begin{array}{ll}
\min & J(X, K)=\operatorname{tr} \int_{0}^{T}\left[Q X(t)+K(t)^{T} R K(t) X(t)\right] d t \\
\text { s.t. } & \quad \dot{X}=(A+B K) X+X(A+B K)^{T} \\
& +(C+D K) X(C+D K)^{T} \\
& X(0)=X_{0} .
\end{array}
$$

Corollary 22. If $K_{*}$ is optimal solution of Problem 21, then there exists a unique $P \in N B V^{n \times n}[0, T]$ to the following constrained GDRE [13]

$$
\begin{gathered}
-\dot{P}=P A+A P^{T}+C^{T} P C+Q \\
-\left(P B+C^{T} P D\right)\left(R+D^{T} P D\right)\left(P B+C^{T} P D\right)^{T}, \\
P(T)=0, \\
\left(R+D^{T} P D\right)\left(R+D^{T} P D\right)^{+}\left(B^{T} P+D^{T} P C\right) \\
=B^{T} P+D^{T} P C, \\
\left(R+D^{T} P D\right) \geq 0 \\
K_{*}=-\left(R+D^{T} P D\right)^{\dagger}\left(B^{T} P+D^{T} P C\right) \\
+Y-\left(R+D^{T} P D\right)^{\dagger}\left(R+D^{T} P D\right) Y,
\end{gathered}
$$

where $Y \in L^{2}\left(0, T ; R^{m \times n}\right)$.

Proof. Because of Problem 21 without the equality constraint, $\lambda=0$, therefore $P(T)=0$. Theorem 20 yields this corollary directly.

\section{Application}

4.1. A Necessary and Sufficient Condition. In Theorem 15, to ensure the uniqueness of $\lambda$ and $P$, let us strengthen the condition $\left(R+D^{T} P D\right) \geq 0$ as $\left(R+D^{T} P D\right)>0$.

Theorem 23. If $K_{*}$ is optimal solution of Problem 1 , then $P \in$ $N B V^{n \times n}[0, T]$ and $\lambda \in R^{1}>0$, such that

$$
\begin{gathered}
-\dot{P}=\left(Q+K_{*}^{T} R K_{*}\right)+P\left(A+B K_{*}\right) \\
+\left(A+B K_{*}\right)^{T} P+\left(C+D K_{*}\right) P\left(C+D K_{*}\right)^{T}, \\
P(T)=\lambda I_{n}, \\
K_{*}^{T} R+P B+\left(C+D K_{*}\right)^{T} P D=0, \\
\lambda[\operatorname{tr} X(T)-c]=0,
\end{gathered}
$$


and if $\left(R+D^{T} P D\right)>0$ hold, then Problem 1 is well posed. The optimal solutions of Problem 1 are

$$
\begin{gathered}
U_{*}=-\left(R+D^{T} P D\right)^{-1}\left(B^{T} P+D^{T} P C\right) x, \\
J_{*}=x_{0}^{T} P(0) x_{0}-\lambda c .
\end{gathered}
$$

Proof. In Remark 3, in order for a minimum point $x^{*}$ of a mathematical programming to satisfy the KKT conditions, we have assumed that $x^{*}$ satisfies LICQ regularity condition. From (19c) of Theorem 15, we obtain

$$
K_{*}\left(R+D^{T} P D\right)=-\left(B^{T} P+D^{T} P C\right) .
$$

Because $\left(R+D^{T} P D\right)>0$, Problem 11 has a unique solution; namely,

$$
K_{*}=-\left(R+D^{T} P D\right)^{-1}\left(B^{T} P+D^{T} P C\right) .
$$

From the relationship between Problems 1 and 11 and by using Itô's formula, we have

$$
J_{*}=V\left(x_{0}\right)=x_{0}^{T} P(0) x_{0}-\lambda c .
$$

4.2. A Dynamic Programming Algorithm. A method for solving the indefinite stochastic linear quadratic (LQ) optimal control problem with unequal terminal state constraint is proposed as follows.

Reconsider the following LQ Problem $1^{\prime}$

$$
\begin{gathered}
d x(t)=[A(t) x(t)+B(t) u(t)] d t \\
+[C(t) x(t)+D(t) u(t)] d w(t), \\
x(s)=y, \\
E\left\{\|x(T)\|^{2}\right\}=E\left\{x^{T}(T) x(T)\right\} \leq c, \\
J\left(x_{0}, u\right)=E\left\{\int_{0}^{T}\left[x^{T}(t) Q(t) x(t)+u^{T}(t) R(t) u(t)\right]\right\} d t \\
V(s, y)=\inf _{u(\cdot) \in U_{\mathrm{ad}}} J(s, y ; u(\cdot)) .
\end{gathered}
$$

Then the backward dynamic programming optimality principle [8] yields the following theorem.
Theorem 24. Let $x_{*}(\cdot)$ and $u_{*}(\cdot)$ be an optimal solution of Problem $1^{\prime}$, then $x_{*}(\cdot)$, and $u_{*}(\cdot)$ satisfy the following Bellman equation:

$$
\begin{gathered}
V(s, y)=\min _{u(\cdot) \in U_{a d}} E\left\{\int _ { s } ^ { s + h } \left[x_{*}^{T}(t) Q(t) x_{*}(t)\right.\right. \\
\left.+u_{*}^{T}(t) R(t) u_{*}(t)\right] d t \\
\left.+V\left(s+h, x_{*}(s+h)\right)\right\} \quad \forall h>0, \\
V(T-h, y)=\min _{u(\cdot) \in U_{a d}} E\left\{\int _ { T - h } ^ { T } \left[x_{*}^{T}(t) Q(t) x_{*}(t)\right.\right. \\
\left.\left.\quad+u_{*}^{T}(t) R(t) u_{*}(t)\right]\right\} d t, \\
E\left\{\|x(T)\|^{2}\right\}=E\left[x^{T}(T) x(T)\right] \leq c .
\end{gathered}
$$

Proof. By applying the backward dynamic programming optimality principle, we obtain recursive relationships between $V\left(s, x_{*}(s)\right)$ and $V\left(s+h, x_{*}(s+h)\right)$ as follows:

$$
\begin{aligned}
V(s, y)=\min _{u(\cdot) \in U_{\mathrm{ad}}} E\left\{\int_{s}^{s+h}\right. & {\left[x_{*}^{T}(t) Q(t) x_{*}(t)\right.} \\
& \left.+u_{*}^{T}(t) R(t) u_{*}(t)\right] d t \\
& \left.+V\left(s+h, x_{*}(s+h)\right)\right\},
\end{aligned}
$$

where $V\left(s, x_{*}(s)\right)$ is the objective of $[s, T]$ and $V\left(s+h, x_{*}(s+\right.$ $h)$ ) of $[s+h, T]$. Let $V\left(s, x_{*}(s)\right)$ be the objective of $[T-h, T]$ and then

$$
\begin{aligned}
V(T-h, y)=\min _{u(\cdot) \in U_{\mathrm{ad}}} E \int_{T-h}^{T} & {\left[x_{*}^{T}(t) Q(t) x_{*}(t)\right.} \\
& \left.+u_{*}^{T}(t) R(t) u_{*}(t)\right] d t
\end{aligned}
$$

Combine the terminal state constraint

$$
\begin{gathered}
E\left\{\|x(T)\|^{2}\right\}=E\left[x^{T}(T) x(T)\right] \leq c, \\
u(t)=K x(t) \quad(K \text { is given by Theorem 20); }
\end{gathered}
$$

thus we can solve the above recursive relations. Furthermore, let $h \rightarrow 0$ generating the dynamic programming algorithm.

Remark 25. The dynamic programming algorithm can be applied to solve the stochastic LQ problem with inequality constraint except for a heavy computation. Nonetheless, it makes more sense in theory. 
Example 26. In Problem 1, let

$$
\begin{gathered}
A=-1, \quad B=1, \quad C=-2, \\
D=0, \quad c=\frac{\left(1+e^{2}\right)^{2}}{4 e^{2}}, \\
x_{0}=1, \quad Q=0, \\
R=1, \quad T=1 .
\end{gathered}
$$

By Theorem 15, we have

$$
\begin{gathered}
-\dot{P}=K_{*}^{2}+2 P\left(-1+K_{*}\right)+4 P, \\
P(1)=\lambda, \\
K_{*}+P=0, \\
\lambda\left[\begin{array}{c}
\left.X(1)-\frac{\left(1+e^{2}\right)^{2}}{4 e^{2}}\right]=0, \\
\lambda \geq 0 .
\end{array}\right.
\end{gathered}
$$

The constraints of Problem 11 are

$$
\begin{gathered}
-\dot{X}=2 X+2 K X, \\
X(0)=1, \\
X(1) \leq \frac{\left(1+e^{2}\right)^{2}}{4 e^{2 t}} .
\end{gathered}
$$

Via a series of computations, we obtain

$$
\begin{aligned}
& X_{*}=\frac{\left(1+e^{2}\right)^{2}}{4 e^{2 t}}, \quad P=\frac{2}{1+e^{2 t}}, \\
& \lambda=\frac{2}{1+e^{2}}, \quad K_{*}=-\frac{2}{1+e^{2 t}} .
\end{aligned}
$$

In Problem 11, the inequality constraint is

$$
G\left(X_{*}(1)\right)=X_{*}(1)-c \leq 0 .
$$

When $T=1$,

$$
X_{*}(1)=\frac{\left(1+e^{2}\right)^{2}}{4 e^{2}}=c .
$$

Hence

$$
G\left(X_{*}(1)\right)=X_{*}(1)-c=0 .
$$

This shows that the optimal solution of Problem 11 satisfies the inequality constraint. In this case, the inequality constraint is a nonactive constraint. Because $\left(R+D^{T} P D\right)=1>0$, using Theorem 23, the optimal control of Problem 1 is

$$
u_{*}(t)=-\frac{2}{1+e^{2 t}} x_{*}(t),
$$

and the optimal value is

$$
V\left(x_{0}\right)=x_{0}^{T} P(0) x_{0}-\lambda c=\frac{1-e^{-2}}{2} .
$$

\section{Conclusion}

We have studied the indefinite stochastic LQ optimal control problem with unequal terminal state constraint, which can be transformed into a hybrid constrained mathematical programming problem. By applying KKT theorem, we have presented a necessary condition for the constrained indefinite stochastic LQ optimal control problem. By adding some conditions, we obtain a necessary and sufficient condition for indefinite stochastic LQ optimal control problem with inequality constraint. We demonstrate that the solvability of the generalized Riccati equation is sufficient for the well posedness of the indefinite LQ problem. Since this kind of LQ control problems can be transformed into a hybrid constrained mathematical programming, we have given a dynamic programming algorithm.

\section{Acknowledgments}

This work is supported by the National Natural Science Foundation of China $(61174078,61170183)$, the Research Fund for the Taishan Scholar Project of Shandong Province of China, the SDUST Research Fund (no. 2011KYTD105), and the State Key Laboratory of Alternate Electrical Power System with Renewable Energy Sources (no. LAPS13018).

\section{References}

[1] R. E. Kalman, "Contributions to the theory of optimal control," Boletín de la Sociedad Matemática Mexicana, vol. 5, no. 2, pp. 102-119, 1960.

[2] W. M. Wonham, "On a matrix Riccati equation of stochastic control," SIAM Journal on Control and Optimization, vol. 6, pp. 681-697, 1968.

[3] C. E. de Souza and M. D. Fragoso, "On the existence of maximal solution for generalized algebraic Riccati equations arising in stochastic control," Systems \& Control Letters, vol. 14, no. 3, pp. 233-239, 1990.

[4] W. Zhang, "Study on generalized algebraic Riccati equation and optimal regulators," Journal of Control Theory and Applications, vol. 20, no. 4, pp. 637-640, 2003.

[5] S. Chen, X. Li, and X. Y. Zhou, "Stochastic linear quadratic regulators with indefinite control weight costs," SIAM Journal on Control and Optimization, vol. 45, no. 6, pp. 1131-1143, 2000.

[6] M. A. Rami and X. Y. Zhou, "Linear matrix inequalities, Riccati equations, and indefinite stochastic linear quadratic controls," IEEE Transactions on Automatic Control, vol. 45, no. 6, pp. 1131$1143,2000$.

[7] C. E. de Souza and M. D. Fragoso, "On the existence of maximal solution for generalized algebraic Riccati equations arising in stochastic control," Systems \& Control Letters, vol. 14, no. 3, pp. 233-239, 1990.

[8] R. Bellman, "On the theory of dynamic programming," Proceedings of the National Academy of Sciences of the United States of America, vol. 38, pp. 716-719, 1952.

[9] D. D. Yao, S. Zhang, and X. Y. Zhou, "Stochastic linear-quadratic control via semidefinite programming," SIAM Journal on Control and Optimization, vol. 40, no. 3, p. 801-823 (electronic), 2001. 
[10] W. Zhang and B.-S. Chen, "On stabilizability and exact observability of stochastic systems with their applications," Automatica, vol. 40, no. 1, pp. 87-94, 2004.

[11] A. Beghi and D. D’Alessandro, "Discrete-time optimal control with control-dependent noise and generalized Riccati difference equations," Automatica, vol. 34, no. 8, pp. 1031-1034, 1998.

[12] D. D. Yao, S. Zhang, and X. Y. Zhou, "Stochastic linearquadratic control via semidefinite programming," SIAM Journal on Control and Optimization, vol. 40, no. 3, pp. 801-823, 2001.

[13] M. Ait Rami, J. B. Moore, and X. Y. Zhou, "Indefinite stochastic linear quadratic control and generalized differential Riccati equation," SIAM Journal on Control and Optimization, vol. 40, no. 4, pp. 1296-1311, 2001.

[14] M. Ait Rami, X. Chen, and X. Y. Zhou, "Discrete-time indefinite LQ control with state and control dependent noises," Journal of Global Optimization, vol. 23, no. 3-4, pp. 245-265, 2002.

[15] Y. Huang, W. Zhang, and H. Zhang, "Infinite horizon linear quadratic optimal control for discrete-time stochastic systems," Asian Journal of Control, vol. 10, no. 5, pp. 608-615, 2008.

[16] G. Wang and $\mathrm{Z}$. Wu, "Stochastic linear quadratic optimal control with partial information and its application to meanvariance hedging problems," in Proceedings of the 27th Chinese Control Conference (CCC '08), pp. 601-605, Yunnan, China, July 2008.

[17] Y. L. Huang and W. H. Zhang, "Study on stochastic linear quadratic optimal control with constraint," Acta Automatica Sinica, vol. 32, no. 2, pp. 246-254, 2006.

[18] H. W. Kuhn and A. W. Tucker, "Nonlinear programming," in Proceedings of the 2nd Berkeley Symposium on Mathematical Statistics and Probability, pp. 481-492, Berkeley University, California Press, 1951.

[19] W. Karush, Minima of functions of several variables with inequalities as side constraints [M.S. thesis], Department of Mathematics, University of Chicago, Chicago, Ill, USA, 1939.

[20] T. H. Kjeldsen, "A contextualized historical analysis of the Kuhn-Tucker theorem in nonlinear programming: the impact of World War II," Historia Mathematica, vol. 27, no. 4, pp. 331361, 2000.

[21] D. G. Luenberger, Optimization by Vector Space Methods, John Wiley \& Sons, New York, NY, USA, 1968.

[22] M. Athans, "The matrix minimum principle," Information and Computation, vol. 11, pp. 592-606, 1967. 


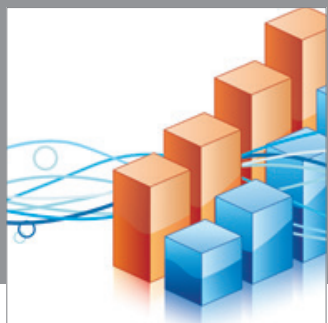

Advances in

Operations Research

mansans

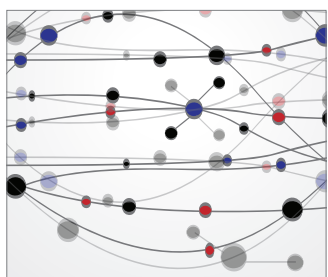

The Scientific World Journal
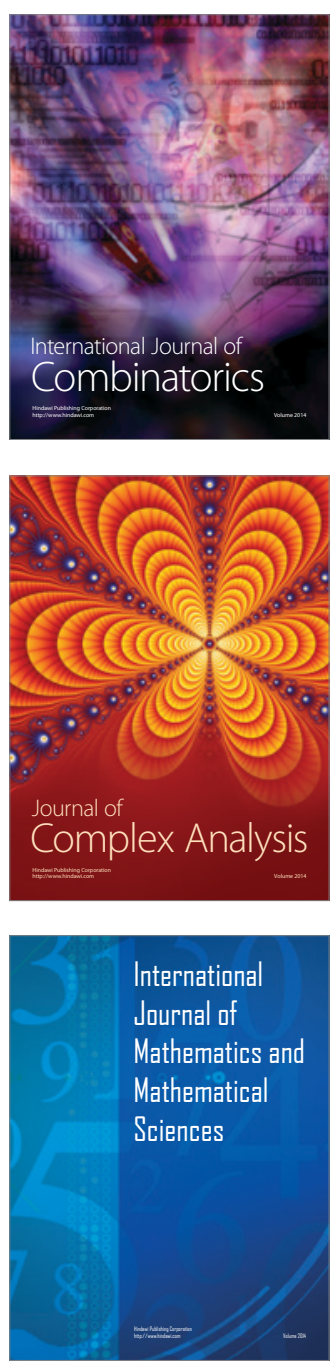
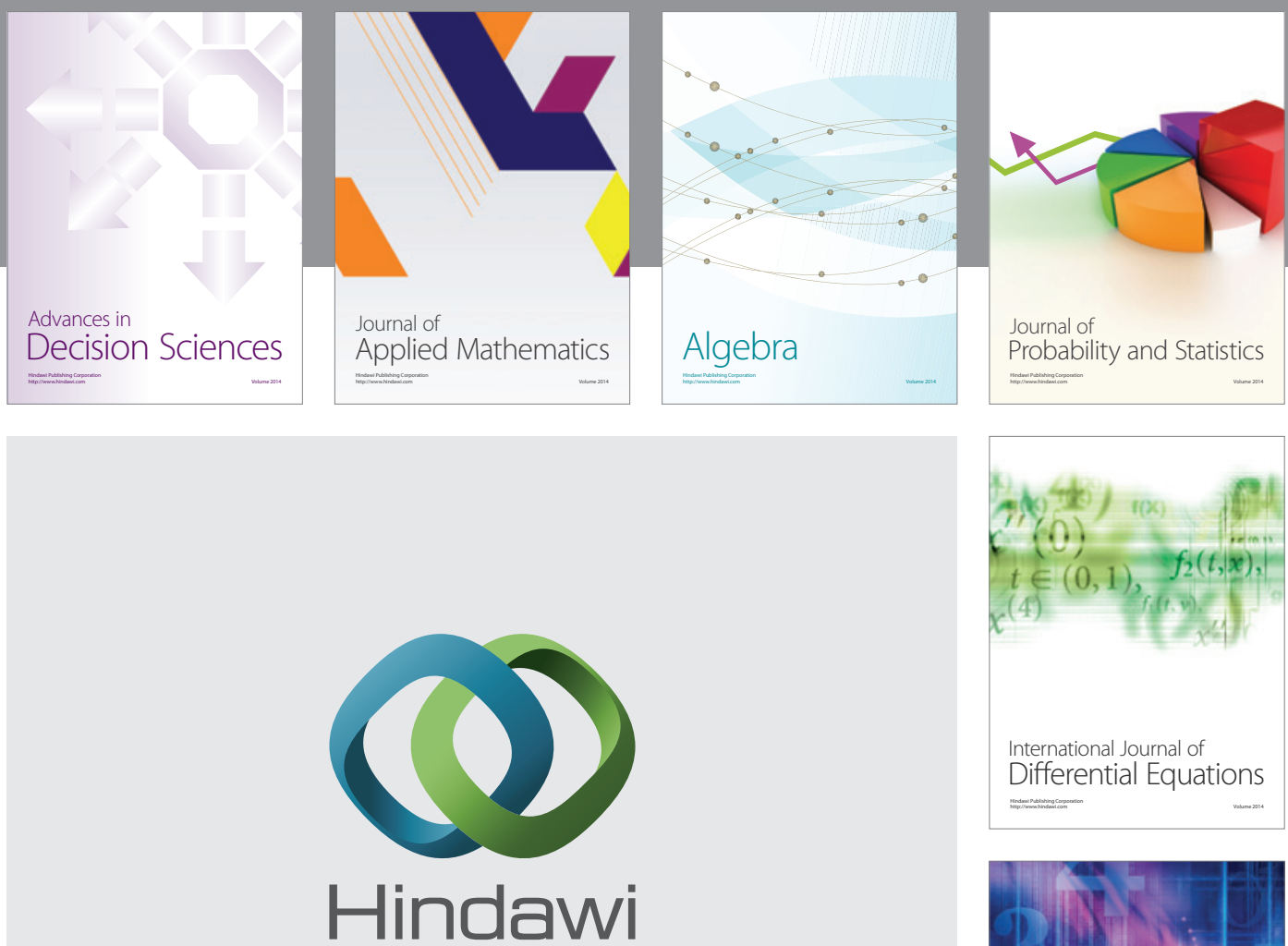

Submit your manuscripts at http://www.hindawi.com
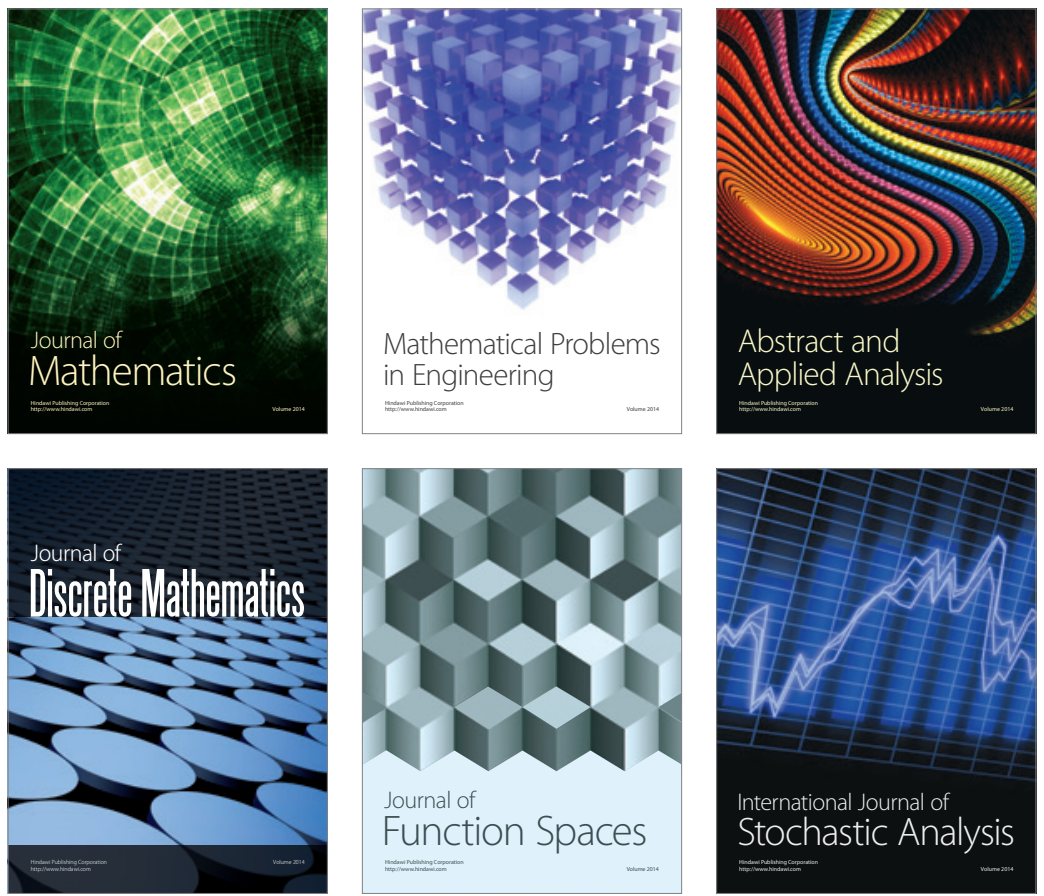

Journal of

Function Spaces

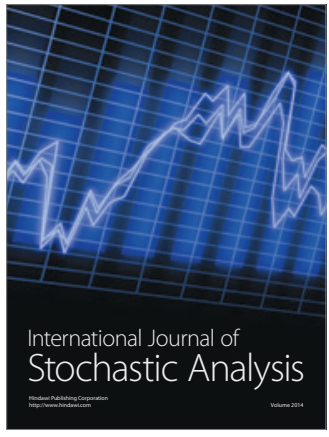

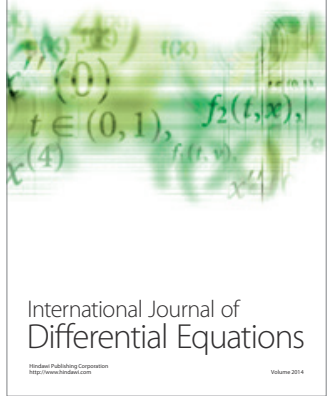
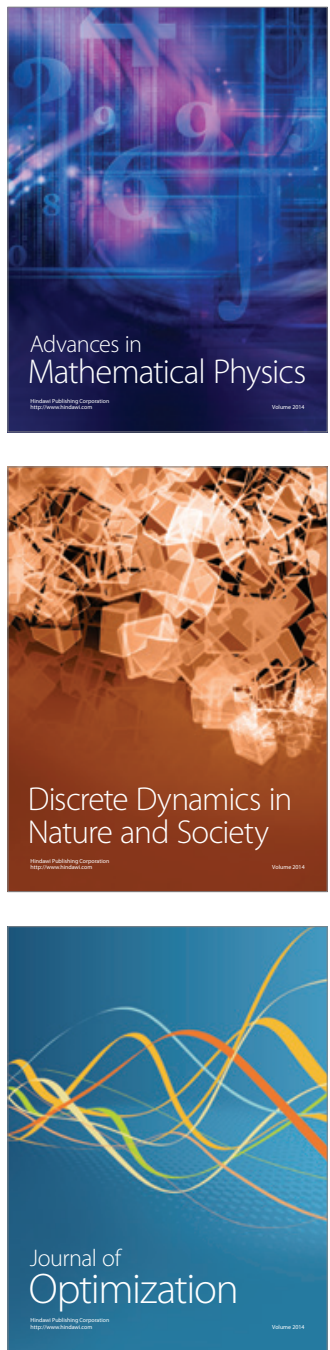